\title{
Associations between Hormonal and Mechanical Factors of Knee Osteoarthritis in Women-A Preliminary Study
}

\author{
David S. Mandeville ${ }^{1}$, Gretchen A. Casazza ${ }^{2}$, Andrea C. Alvarez ${ }^{3}$, Julia Sheremet ${ }^{3}$, Brandee L. Waite ${ }^{2}$, \\ Brian A. Davis ${ }^{2}$
}

${ }^{1}$ Department of Kinesiology and Health Science, California State University, Sacramento, USA; ${ }^{2}$ Sports Medicine Program, University of California, Davis Health System, Sacramento, USA; ${ }^{3}$ Department of Physical Therapy, California State University, Sacramento, USA.

Email: dmandeville@csus.edu

Received February $14^{\text {th }}, 2013$; revised March 23 ${ }^{\text {rd }}, 2013$; accepted April $1^{\text {st }}, 2013$

Copyright (C) 2013 David S. Mandeville et al. This is an open access article distributed under the Creative Commons Attribution License, which permits unrestricted use, distribution, and reproduction in any medium, provided the original work is properly cited.

\begin{abstract}
The purpose of this study was to determine the associations between urinary estradiol $\left(\mathrm{E}_{2}\right)$ metabolite concentration and medial knee loading with radiographic disease severity in middle aged women with initial stage knee osteoarthritis (OA). Women presenting with knee pain were recruited into a cross-sectional correlation study (KOA, $\mathrm{n}=9$, age $=52 \pm$ 4 yrs). Self report menstrual history, the Modified Baecke Questionnaire and the Knee Injury and Osteoarthritis Outcome Score (KOOS) subjective data were collected. A fasting blood sample (follicle stimulating hormone (FSH) and Tumor Necrosis Factor- $\alpha$ (TNF- $\alpha$ )), and urine catch (16 $\alpha$-hydroxyestrone and 2-hydroxyestrone) were collected. Gait analysis using an 8-camera motion analysis system assessed internal knee varus moment and foot progression angle. Pearson Product moments tested for associations between urinary 16 $\alpha$-hydroxyestrone and 2-hydroxyestrone, TNF- $\alpha$, medial knee loading, and radiographic disease severity (Kellgren/Lawrence (K/L) radiographic score). Significant correlations were found within the hormonal biomarkers $(\mathrm{r}=0.94, p<0.001)$ and within the biomechanical markers $(\mathrm{r}=$ $0.76, p<0.02)$, but not between hormonal and biomechanical variables $(\mathrm{r}=0.39, p=0.31)$. No correlations were found for radiographic disease severity or TNF- $\alpha$. The lack of association between hormonal and biomechanical variables could be due to large variability of the $\mathrm{E}_{2}$ metabolites seen in the menopause transition and the limited structural changes of initial staged knee OA.
\end{abstract}

Keywords: Knee Osteoarthritis; 2-Hydroxyestrone; Follicle Stimulating Hormone; Gait Analysis; Medial Knee Loading; Tumor Necrosis Factor- $\alpha$

\section{Introduction}

One of the leading causes of disability in the elderly is the pain associated with knee osteoarthritis (OA) [1]. Chronic pain from knee OA can decrease physical activity, increasing the risk for: chronic disease, decreased functional independence and diminished quality of life [2]. Within the next 30 years, it is predicted that $25 \%$ of adults will have arthritis and at least $2 / 3$ of these patients will be women [3]. In spite of the increased prevalence of knee $\mathrm{OA}$ in women, there has been limited investigation into the factors underlying the sex disparity of knee OA [2]. However, the increased incidence of knee OA around the age of menopause suggests that estrogen deprivation may play a role in the development of knee OA in women. Urinary estradiol $\left(\mathrm{E}_{2}\right)$ metabolites were shown to be associated with the incidence of knee OA in perimenopausal women, possibly due to regulation of arachidonic acid metabolism [4]. Additionally, greater knee loading across the medial compartment during walking has been shown to predict medial knee OA progression [5-7]. It may be possible to ascertain the influence of $E_{2}$ in the etiology of knee OA by tracking its association across time to the established pathomechanic marker of medial knee loading.

Estradiol is the major bioactive estrogen and is produced mainly by the ovaries in non-pregnant women, but small amounts are also produced by adipose cells and the adrenal cortex [8]. Estradiol is secreted into the blood stream and estrogenic responses at the tissue level are mediated by $\mathrm{E}_{2}$-receptor complexes (ER $\alpha \& \mathrm{ER} \beta$ ) [9]. Estradiol is converted to estrone $\left(E_{1}\right)$, after which further enzymatic catabolism produces the 2-hydroxyestrone and 16 $\alpha$ - 
hydroxyestrone metabolites [4]. Recent evidence suggests that the $\mathrm{E}_{2}$ reductions occurring at menopause may mediate the degenerative changes of articular cartilage at the knee in women [4]. Estradiol receptors have been identified in normal and osteoarthritic cartilage, indicating that cartilage is sensitive to $E_{2}$ [10-12]. Thus, as serum $E_{2}$ concentrations decrease with menopause, a receptor-based mechanism may decrease the anabolic chondrocyte activity typical of premenopause, adversely affecting cartilage homeostasis [13].

Circulating concentrations of proinflammatory cytokines are higher in persons with osteoarthritis and are associated with radiographic knee OA progression $[14,15]$. Evidence suggests that declining estrogen byproducts may allow for increased expression of proinflammatory metabolism [4]. Mechanical sheer stress has also been shown to interfere with cartilage homeostasis via stimulation of proinflammatory cytokines [16]. In vitro, proinflammatory cytokines have been shown to compromise the load bearing properties of cartilage via decreased collagen synthesis, proteoglycan loss, and ultimately decreased water retention within the extra-cellular matrix [17]. As a result of an inflammatory interruption of chondrocyte homeostasis, articular cartilage may lose the ability to adapt to the cyclic loading of activities of daily living, leading to fibrillation and ultimately destruction [18]. As the etiology of knee OA has been described as involving local mechanical factors occurring within systemic susceptibility [19], it is plausible that cartilage destruction occurs along an integrated hormonal and biomechanical pathway. In such a model, decreased $\mathrm{E}_{2}$ metabolite concentration would predispose articular cartilage to catabolism where knee loading would then drive cartilage destruction and bony hypertrophy via joint overload. The effects of both factors would be linked via increased circulating inflammatory cytokines. However, no studies have assessed both the hormonal and mechanical factors concomitantly for women with knee OA in the menopause transition.

If an association exists between hormonal and mechanical factors in the progression of knee OA, this information could be used to implement early diagnostic and conservative intervention paradigms, designed to mitigate disease progression and sparing women irreversible joint damage. As women represent a growing majority of knee OA patients, efforts to limit disease progression and to preserve knee joint viability will address a substantial societal need. The benefits of preventing knee OA are multiplicative, as women with increased functional independence are capable of controlling obesity, a targeted modifiable risk factor of cardiovascular disease and diabetes [20]. Such preventive intervention will also allay the enormous economic burden of total knee replacement surgery in the coming decades [21]. A need exists for an interdisciplinary analysis that describes the role of these factors in the disease progression and describes a possible mechanism whereby they converge. The purpose of this study was to determine the associations between urinary $\mathrm{E}_{2}$ metabolite concentration and medial knee loading with the radiographic knee OA severity in women. Additionally, the relationship of circulating proinflammatory cytokines to both $\mathrm{E}_{2}$ metabolites and medial knee loading was assessed. It was predicted that $\mathrm{E}_{2}$ would be negatively related to medial knee loading.

\section{Materials and Methods}

Women presenting with knee pain were recruited from the UC Davis Sports Medicine Clinic into an initial-staged medial knee OA group (KOA, $\mathrm{n}=9$, age $=52 \pm 4$ yrs; Table 1). Subjects were diagnosed with initial staged knee OA based on bilateral anteroposterior $(\mathrm{A} / \mathrm{P})$ weightbearing knee radiographs. The 2 study physicians independently evaluated the radiographs (blinded for subject name or other identifiers) for the presence and severity of specific radiographic features of OA, including osteophytes, joint deformity, sclerosis and joint space narrowing. Overall evidence of OA in each knee was graded on a scale of 0 - IV using the Kellgren and Lawrence scoring system with $0=$ normal, $\mathrm{I}=$ doubtful $\mathrm{OA}, \mathrm{II}=$ minimal OA, III = moderate OA, and IV = severe OA [22]. Women having knees with a score of III or IV at baseline were excluded. Additional subject inclusion criteria included: age 45 - 55 years, medically stable, and nonsmoking. Exclusion criteria included: no hormone replacement therapy in the last 2 years, no prior ovariectomy, no knee or hip replacement surgery. All subjects were informed of the risks and benefits of the experiment and provided informed, voluntary consent approved by the UC Davis Institutional Review Board.

Subjective assessment occurred following a 1-week washout period for anti-inflammatory medications. Subjects completed a health history questionnaire, a Modified Baecke Questionnaire and the Knee Injury and Osteoarthritis Outcome Score (KOOS). As part of the health history, subjects reported menstrual cycle status as days since last period. The Modified Baecke Questionnaire has

Table 1. Mean (SD) subject demographics of nine women with initial staged knee OA in the menopause transition.

\begin{tabular}{|c|c|c|c|c|c|}
\hline & Age (years) & Height (m) & Mass (kg) & BMI $\left(\mathrm{kg} / \mathrm{m}^{2}\right)$ & $\mathrm{FSH}(\mathrm{mIU} / \mathrm{ml})$ \\
\hline Mean (SD) Range & $\begin{array}{c}52(4) \\
(46-57)\end{array}$ & $\begin{array}{c}1.67(0.05) \\
(1.64-1.79)\end{array}$ & $\begin{array}{c}76.6(16.0) \\
(59.8-103.2)\end{array}$ & $\begin{array}{c}27.5(5.7) \\
(22.6-38.4)\end{array}$ & $\begin{array}{c}64.6(41.2) \\
(14.2-121.4)\end{array}$ \\
\hline
\end{tabular}


been previously validated for assessing physical activity levels [23]. The KOOS (Likert Scale) has been validated as moderate to high responsiveness when used to assess symptoms, function, pain and quality of life for subjects treated for knee osteoarthritis [24].

Subjects were asked to refrain from anti-inflammatory medications for 4 - 7 days prior to biomarker testing. Circulating Follicle Stimulating Hormone (FSH) and Tumor Necrosis Factor- $\alpha$ (TNF- $\alpha$ ) concentrations were tested with $5 \mathrm{ml}$ of serum sampled using a standard venipuncture protocol at UC Davis Sports Medicine. Serum samples were sent immediately to UC Davis Clinical Laboratory, Sacramento, CA for cell separation, freezing and FSH analysis. Plasma samples were tested for TNF- $\alpha$ level using quantitative multiplex bead assays (ARUP Laboratories, Salt Lake City, UT). Urinary 16 $\alpha$-hydroxyestrone and 2-hydroxyestrone concentrations were assayed from urine collected at the phlebotomy appointment. Urine samples were centrifuged and acidified with ascorbic acid prior to storage at $-80^{\circ} \mathrm{F}$ until assayed using the Estramet 2/16 competitive, solid phase immunoassay (Immuna Care Corp, PA USA) at the Clinical and Epidemiological Research Laboratory, Children's Hospital, Boston, MA.

The knee varus moment was tested by gait analysis of the subject's level walking using an 8-camera Vicon motion capture system $(100 \mathrm{~Hz}$, Oxford Metrics, UK) and a force platform (1000 Hz, AMTI, Newton, MA, USA) at the Biomechanics Laboratory, California State University, Sacramento. Subjects wore their own walking shoes, comfortable clothing and 34 retroreflective markers of a whole body model (Plug-in-Gait, marker diameter $=13$ $\mathrm{mm})$. Subjects were instructed to walk at their preferred walking speed along a $10-\mathrm{m}$ walkway. The mean of 3 - 5 trials was used for further analysis. The second peak of the internal knee varus moment and the concomitant foot progression angle during stance were used for further analysis.

The intra-class correlation coefficient was used to assess the level of agreement for the radiographic Kellgren/Lawrence ratings ( $\alpha=0.05$, SPSS Inc., Chicago, IL). Pearson product moments $(\alpha=0.05$, SPSS Inc., Chicago, IL) were used to test for significance of the associations bet- ween the dependent variables: subjective menstrual status and symptoms, urinary 2-hydroxyestrone/16 $\alpha$-hy-droxyestrone, circulating TNF- $\alpha$, circulating FSH, K/L rating, the second peak knee varus moment and concomitant foot progression angle.

\section{Results}

Women were found to be at diverse stages of follicular activity, as indicated by the variability of the mean circulating FSH level, $64.5 \pm 41.1 \mathrm{mIU} / \mathrm{ml}$ (Table 1). The Kellgren/Lawrence knee osteoarthritis ratings of the involved limb were found to have significant agreement (ICC $=0.87, p=0.001)$ and the mean value of the 2 ratings was used for further analysis. All subjects had initial staged knee $\mathrm{OA}$, as indicated by the $\mathrm{K} / \mathrm{L}$ rating of $1.59 \pm$ 0.80 (Table 2). The mean urinary $\mathrm{E}_{2}$ metabolite level for 2-hydroxyestrone was found to be: $22.38 \pm 31.32 \mathrm{pg} / \mathrm{ml}$, while $16 \alpha$-hydroxyestrone was: $19.7 \pm 22.47 \mathrm{pg} / \mathrm{ml}$ (Table 2). Mean circulating TNF- $\alpha$ values were $26.22 \mathrm{pg} / \mathrm{ml}$ \pm 49.36 (Table 2). The mean 2 nd peak knee varus moment was found to be $3.08 \% \pm 1.11 \% \mathrm{BWBH}$; while the mean concomitant foot progression angle was $18.24^{\circ} \pm 4^{\circ}$ (Table 2).

No significant correlations were found between the urinary $\mathrm{E}_{2}$ metabolites and the biomechanical markers (Table 2, Figure 1). A significant positive correlation was seen between 2-Hydroxyestrone and 16 $\alpha$-Hydroxyestrone $(\mathrm{r}=0.937 ; p<0.0001$, Table 2, Figure 1). Biomechanical markers (knee varus moment and foot progression angle) were found to have a significant negative correlation ( $\mathrm{r}=-0.762 ; p=0.017$, Table 2, Figure 1). Circulating FSH was positively correlated to days since last menstrual period $(\mathrm{r}=0.781 ; p=0.02$, Table 3$)$. Circulating FSH values were also negatively correlated to KOOS quality of life score $(\mathrm{r}=-0.809 ; p=0.008$, Table 3). The $\mathrm{K} / \mathrm{L}$ values were positively correlated to KOOS pain scores; $(\mathrm{r}=-0.865 ; p=0.003$, Table 3); and negatively correlated to KOOS ADL's score $(\mathrm{r}=-0.732$; $p=0.025$ ), and negatively correlated to BACKE work index score $(\mathrm{r}=-0.739 ; p=0.036$, Table 3).

\section{Discussion}

The purpose of this pilot study was to begin testing the

Table 2. Associations between biomarkers (hormonal and inflammatory) and biomechanical markers (knee varus moment) of disease progression for nine women with initial stage knee $\mathrm{OA}$ in the menopause transition.

\begin{tabular}{|c|c|c|c|c|c|c|}
\hline & $\begin{array}{c}\mathrm{K} / \mathrm{L}(0=\text { no KOA; } \\
\mathrm{IV}=\text { end stage } \\
\mathrm{KOA})\end{array}$ & $\begin{array}{l}\mathrm{TNF}-\alpha \\
(\mathrm{pg} / \mathrm{ml})\end{array}$ & $\begin{array}{l}\text { 2-Hydroxyestrone } \\
\text { (pg/mL) }\end{array}$ & $\begin{array}{c}16 \alpha \text {-Hydroxyestrone } \\
(\mathrm{pg} / \mathrm{mL})\end{array}$ & $\begin{array}{l}\text { Knee Varus } \\
\text { Moment } \\
(\% \mathrm{BWBH})\end{array}$ & $\begin{array}{l}\text { Foot Progression } \\
\text { Angle (Degrees) }\end{array}$ \\
\hline Mean (SD) & $1.59(0.8)$ & $26.2(49.4)$ & $23.3(31.3)$ & $19.7(22.5)$ & $3.1(1.1)$ & $18.24(4.0)$ \\
\hline \multirow[t]{2}{*}{ Range } & $(0-2.5)$ & $(4-151)$ & $(0.6-100.6)$ & $(4.8-77.0)$ & $(1.7-5.4)$ & $(15.6-25.7)$ \\
\hline & none & none & $*$ & $*$ & $* *$ & $* *$ \\
\hline
\end{tabular}

${ }^{*}$ Significant correlation 2-Hydroxyestrone and $16 \alpha$-Hydroxyestrone; $p<0.0001 .{ }^{* *}$ Significant correlation 2 nd peak knee varus moment and concomitant foot progression angle; $p=0.02$. 
Table 3. Associations between subjective data (KOOS, BACKE, and menstrual cycle status): vs. Follicle Stimulating Hormone (FSH); and vs. disease severity (K/L scale) for women with initial stage knee OA in the menopause transition.

\begin{tabular}{|c|c|c|c|c|c|}
\hline & $\begin{array}{c}\text { KOOS Pain } \\
(100-0 \text { pts, } \\
0=\text { worst })\end{array}$ & $\begin{array}{c}\text { KOOS ADL } \\
(100-0 \text { pts } \\
0=\text { worst })\end{array}$ & $\begin{array}{c}\text { KOOS QOL } \\
(100-0 \text { pts, } \\
0=\text { worst })\end{array}$ & $\begin{array}{c}\text { Days since Last } \\
\text { Menstrual Cycle } \\
\text { (Days) }\end{array}$ & $\begin{array}{l}\text { BAECKE Work Index } \\
\text { ( } \uparrow \text { value }=\uparrow \text { function) }\end{array}$ \\
\hline Mean (SD) & $70.05(12.01)$ & 78.27 (12.98) & $36.8(20.11)$ & 1414 (1155) & $2.39(0.54)$ \\
\hline FSH (mIU/ml) & none & none & $\begin{array}{l}\mathrm{r}=-809 \\
p=0.008\end{array}$ & $\begin{array}{l}\mathrm{r}=0.781 \\
p=0.022\end{array}$ & none \\
\hline $\begin{array}{c}\mathrm{K} / \mathrm{L} \\
\text { (stage } 0 \text { - IV) }\end{array}$ & $\begin{array}{c}\mathrm{r}=-0.865 \\
p=0.003\end{array}$ & $\begin{array}{l}\mathrm{r}=-0.732 \\
p=0.025\end{array}$ & none & none & $\begin{array}{c}\mathrm{r}=-0.739 \\
p=0.036\end{array}$ \\
\hline
\end{tabular}
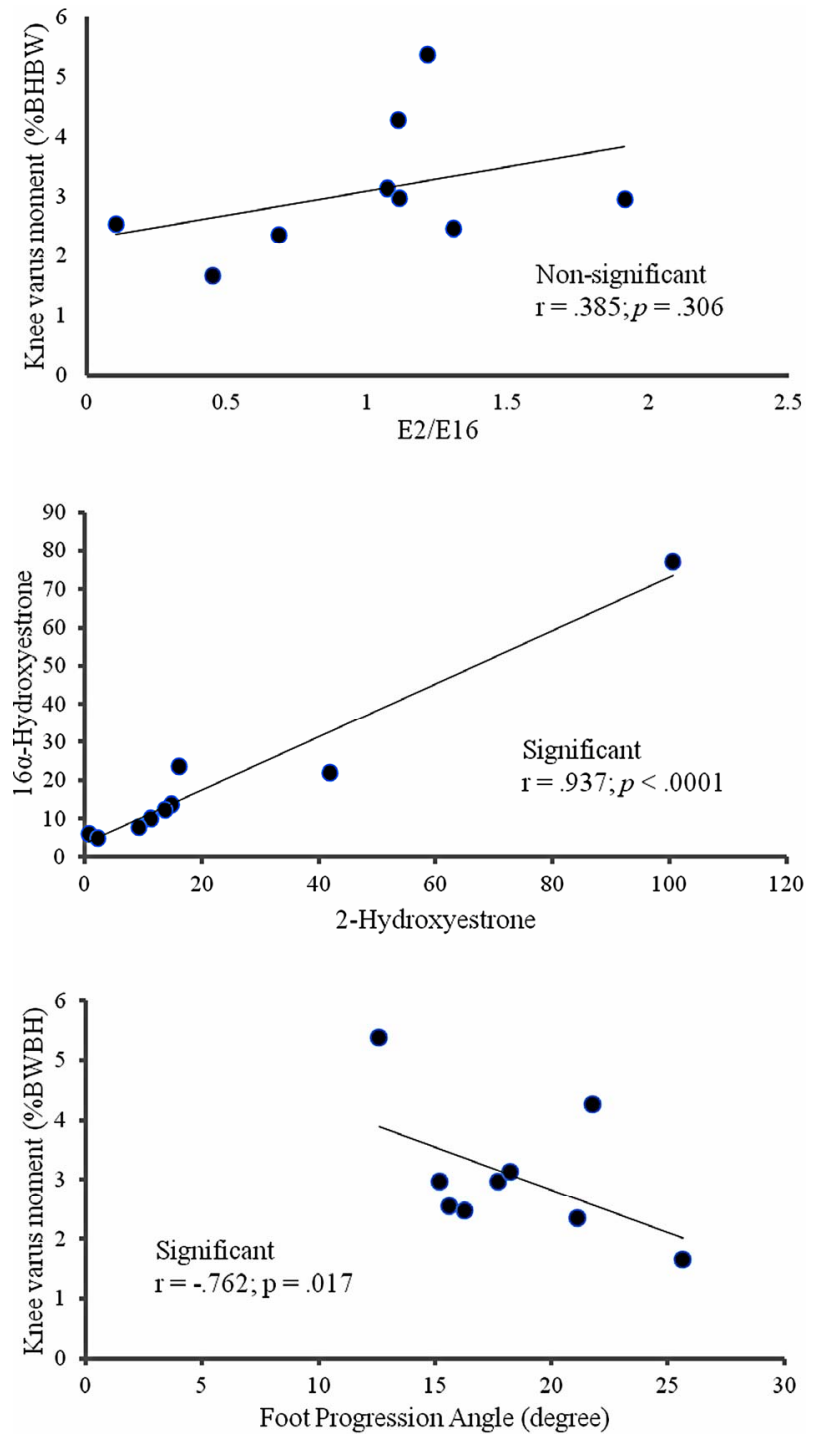

Figure 1. Scatter plots of the associations between: knee varus moment and estradiol metabolites (A); knee varus moment and foot progression angle (B); 16 $\alpha$-hydroxyestrone and 2-hydroxyestrone (C) indicating the hormonal outlier for women with knee $\mathrm{OA}$ in the menopause transition.

hypothesis that hormonal biomarkers are related to known biomechanical markers of knee OA progression.
This report shows the initial findings of a proposed follow-up study in which knee OA and menopause progression would be assessed using imaging, biomarkers, and biomechanical markers. The initial results do not support the hypothesized association between urinary estradiol $\left(\mathrm{E}_{2}\right)$ metabolite concentration and medial knee loading for women with knee OA in the menopause transition. This may be due to the initial stages of both knee $\mathrm{OA}$ and the menopause transition for these subjects in which neither the hormonal nor mechanical makers have become well-defined.

The menopausal status of the women with initial staged knee OA in this study was characterized by both the self reported days since last menstrual period and by the circulating FSH level. The variance of the self-report menstrual status value $(1414.1 \pm 1081$ days $)$ suggests that this value, based on vague time estimates, may not be an accurate indicator of the menopausal transition. The mean circulating FSH values $(64.6 \pm 41.2 \mathrm{mUI} / \mathrm{ml})$ also showed large variability which may undermine the reliability of this indirect marker of follicu lar activity to specify menstrual status. Burger et al. (2002) have previously reported that both circulating $\mathrm{FSH}$ and $\mathrm{E}_{2}$ measures have limited usefulness for categorizing menstrual status for women in the menstrual transition, a time in which generalizations are reported to be unsafe due to marked hormonal fluctuations [25]. Hee et al. (1993), reported FSH variability across the menstrual cycle for individual women $(\mathrm{n}=3$, age $=45-46$ years $)$ and found that values ranged from normal reproductive levels to postmenopausal levels depending on when in the cycle the FSH was assessed [26]. We attempted to collect FSH consistently during the early follicular phase, when possible.

Though it is difficult to ascertain the menstrual status of the women in this study in menopausal transition, their mean FSH value $(64.6 \mathrm{mIU} / \mathrm{ml})$ was similar to the mean value previously reported for a group of women categorized prospectively as late perimenopausal, with no menses in the preceding 3 - 11 months $(72.2 \mathrm{mIU} / \mathrm{ml})$ [25]. Additionally, the urinary $\mathrm{E}_{2}$ metabolite levels (2-hydroxyestrone $=22.38 \pm 31.32 \mathrm{pg} / \mathrm{ml} ; 16 \alpha$-hydroxyestrone $=19.7 \pm 22.47 \mathrm{pg} / \mathrm{ml}$ ) were larger than those reported by 
Sowers et al., (2006) for pre and perimenopausal women with incident knee OA, (2-hydroxyestrone $=11.3 \pm 8.2$ $\mathrm{pg} / \mathrm{ml} ; 16 \alpha$-hydroxyestrone $=7.8 \pm 5.5 \mathrm{pg} / \mathrm{ml}$ ) [4]. Thus, it is likely that these women were nearing the end of the menopausal transition and entering menopause. At that time, the circulating TNF- $\alpha$ values $(26.22 \mathrm{pg} / \mathrm{ml} \pm 49.36)$ were greater than the healthy range $(0-22 \mathrm{pg} / \mathrm{ml})$. Furthermore, the women had initial staged knee OA walked with a mean knee varus moment $(3.08 \pm 1.11 \% \mathrm{BWBH})$ that was within the range of values previously reported for end staged knee OA $(4.07 \pm 0.38 \% \mathrm{BWBH})$ and controls $(3.07 \pm 0.30 \% \mathrm{BWBH})[5]$.

There was also no correlation between $\mathrm{E}_{2}$ metabolites and radiographic knee $\mathrm{OA}$ severity $(\mathrm{K} / \mathrm{L}$ score) for women with knee OA at the age when menopause begins. This result supports the findings of de Klerk et al. (2009), whose systematic review of the literature reporting on the association between female hormones and OA of the hand, hip and knee indicated inconclusive evidence supporting the relationship. The authors suggest that the relationship, if it does exist, may be too complex for the current methodological constraints which may not be able to parse out the effect of hormonal factors from other risk factors [27]. However, Sowers et al. (2006) have reported that perimenopausal women with $\mathrm{E}_{2}$ metabolites in the lowest quartile had increased risk for developing radiographical knee OA [4]. In a further study of perimenopausal women, Sowers et al. (2009) report that longitudinal changes of biomarkers of cartilage and collagen catabolism (serum cartilage oligomeric matrix protein and urinary Type II collagen telopeptides) were associated with knee OA progression, but had modest sensitivity in predicting incident knee OA [28]. Thus, an array of local catabolic and systemic endocrine biomarkers may be needed to predict knee OA disease progression.

From the mechanical perspective, Cerejo et al. (2002) found the effect of knee varus alignment, a mechanical factor influencing the knee varus moment, to be more pronounced for later staged knee OA [29]. The authors suggest that the effect of the mechanical factor was confounded by the vulnerability of the cartilage, which was more susceptible to damage in the moderate to late stages of the disease. Although the literature presents equivocal findings for the role of both the hormonal and mechanical factors for incident and early staged knee OA, a clearer picture may be obtained longitudinally when both the hormonal and mechanical changes of menopause and knee OA become salient. As the primary costs associated with knee OA are due to the disease progression [19], further understanding of the association of the hormonal factors to the established mechanical factor of disease progression is required for attempts to mitigate disease progression.

Several significant associations indicate that the study was powered sufficiently to avoid type II error within the hormonal biomarkers (between 2-Hydroxyestrone and $16-\alpha$-Hydroxyestrone) and within the mechanical makers (between knee varus moment and foot progression angle). Additional relationships indicated that as $\mathrm{K} / \mathrm{L}$ knee $\mathrm{OA}$ rating score (disease severity) increased, pain became more pronounced and functional level (ADLs) decreased, as would be expected. As FSH levels increased, the number of days since last menstrual cycle increased. Further, as FSH values increased, quality of life values decreased, though no association was found between $\mathrm{K} / \mathrm{L}$ rating of disease severity and KOOS quality of life. This finding may suggest that menopausal changes affecting quality of life, such as hot flashes, may be more problematic for maintaining quality of life than the articular changes of initial staged knee OA.

The results of this preliminary cross sectional study should be interpreted with caution due to the limited sample size. Additionally, without the outcomes from follow-up testing, this data does not assess the role of a hormonal biomarker of knee OA progression with respect to an established pathomechanical marker. Due to the large hormonal fluxes present in women in the menopause transition, associations for the biomarkers were likely masked by the large variability seen for FSH $(64.59 \pm 41.22), 2$-hydroxyestrone $(23.38 \pm 31.32)$ and $16 \alpha$-hydroxyestrone $(19.70 \pm 22.47)$. This variability may diminish at the follow-up testing as the biomarker concentrations stabilize. The findings are also limited by the comparison of systemic biomarkers to local biomechanical markers. Future studies could assay and correlate local biomarkers from the knee attained via aspiration at the time of viscosupplementation. This study is also limited by a lack of a control comparison group. Future longitudinal studies are needed to compare women with knee OA to aged-matched healthy women with both groups in the menstrual transition so as to isolate the effect of knee OA from the effect of menopause.

The results of this interdisciplinary study did not support the hypothesized association between urinary $E_{2}$ metabolite concentration and medial knee loading for women with initial stage knee OA in the menopause transition. This finding may be due to the large variability of the $E_{2}$ metabolites seen in the menopause transition, as well as, the limited structural changes of initial staged knee OA. Future follow-up studies are needed to elucidate the relationships of hormonal and mechanical markers across knee OA progression for women in the menopause transition.

\section{REFERENCES}

[1] S. T. Sutbeyaz, N. Sezer, B. F. Koseoglu, F. Ibrahimoglu and D. Tekin, "Influence of Knee Osteoarthritis on Exer- 
cise Capacity and Quality of Life in Obese Adults," Obesity, Vol. 15, No. 8, 2007, pp. 2071-2076.

doi:10.1038/oby.2007.246

[2] J. A. Buckwalter and D. R. Lappin, "The Disproportionate Impact of Chronic Arthralgia and Arthritis among Women," Clinical Orthopaedics and Related Research, Vol. 372, 2000, pp. 159-168. doi:10.1097/00003086-200003000-00018

[3] J. M. Hootman and C. G. Helmick, "Projections of US Prevalence of Arthritis and Associated Activity Limitations," Arthritis \& Rheumatism, Vol. 54, No. 1, 2006, pp. 226-229. doi:10.1002/art.21562

[4] M. R. Sowers, D. McConnell, M. Jannausch, A. G. Buyuktur, M. Hochberg and D. A. Jamadar, "Estradiol and Its Metabolites and Their Association with Knee Osteoarthritis," Arthritis \& Rheumatism, Vol. 54, No. 8, 2006, pp. 2481-2487. doi:10.1002/art.22005

[5] T. P. Andriacchi and A. Mundermann, "The Role of Ambulatory Mechanics in the Initiation and Progression of Knee Osteoarthritis," Current Opinion in Rheumatology, Vol. 18, No. 5, 2006, pp. 514-518. doi:10.1097/01.bor.0000240365.16842.4e

[6] T. M. Griffin and F. Guilak, "The Role of Mechanical Loading in the Onset and Progression of Osteoarthritis," Exercise \& Sport Sciences Reviews, Vol. 33, No. 4, 2005, pp. 195-200. doi:10.1097/00003677-200510000-00008

[7] T. Miyazaki, M. Wada, H. Kawahara, M. Sato, S. Baba and S. Shimada, "Dynamic Load at Baseline Can Predict Radiographic Disease Progression in Medial Compartment Knee Osteoarthritis," Annals of the Rheumatic Diseases, Vol. 61, No. 7, 2002, pp. 617-622. doi:10.1136/ard.61.7.617

[8] G. E. Abraham, W. D. Odell and R. S. Swerdloff, "Simultaneous Radioimmunoassay of Plasma FSH, LH, Progesterone, 17-Hydroxyprogesterone, and Estradiol-17Beta during the Menstrual Cycle," The Journal of Clinical Endocrinology \& Metabolism, Vol. 34, No. 2, 1972, pp. 312-318. doi:10.1210/jcem-34-2-312

[9] K. P. McNatty, D. T. Baird, A. Bolton, P. Chambers, C. S. Corker and H. McLean, "Concentration of Oestrogens and Androgens in Human Ovarian Venous Plasma and Follicular Fluid throughout the Menstrual Cycle," Journal of Endocrinology, Vol. 71, No. 1, 1976, pp. 77-85. doi:10.1677/joe.0.0710077

[10] R. S. Richmond, C. S. Carlson, T. C. Register, G. Shanker and R. F. Loeser, "Functional Estrogen Receptors in Adult Articular Cartilage: Estrogen Replacement Therapy Increases Chondrocyte Synthesis of Proteoglycans and Insulin-Like Growth Factor Binding Protein 2," Arthritis \& Rheumatism, Vol. 43, No. 9, 2000, pp. 2081-2090. doi:10.1002/1529-0131(200009)43:9<2081::AID-ANR20 >3.0.CO;2-I

[11] C. L. Tsai and T. K. Liu, "Osteoarthritis in Women: Its Relationship to Estrogen and Current Trends," Life Sciences, Vol. 50, No. 23, 1992, pp. 1739-1744. doi:10.1016/0024-3205(92)90056-U

[12] T. Ushiyama, H. Ueyama, K. Inoue, I. Ohkubo and S. Hukuda, "Expression of Genes for Estrogen Receptors Alpha and Beta in Human Articular Chondrocytes," Os- teoarthritis and Cartilage, Vol. 7, No. 6, 1999, pp. 560566. doi:10.1053/joca. 1999.0260

[13] A. Breu, B. Sprinzing, K. Merkl, V. Bechmann, R. Kujat, Z. Jenei-Lanzl, et al., "Estrogen Reduces Cellular Aging in Human Mesenchymal Stem Cells and Chrondrocytes," Journal of Orthopaedic Research, Vol. 29, No. 10, 2011, pp. 1563-1571. doi:10.1002/jor.21424

[14] M. Sharif, L. Shepstone, C. J. Elson, P. A. Dieppe and J. R. Kirwan, "Increased Serum C Reactive Protein May Reflect Events that Precede Radiographic Progression in Osteoarthritis of the Knee," Annals of the Rheumatic Diseases, Vol. 59, No. 1, 2000, pp. 71-74. doi:10.1136/ard.59.1.71

[15] T. D. Spector, D. Nandra, D. J. Hart and D. V. Doyle, "Is Hormone Replacment Therapy Protective for Hand and Knee Osteoarthritis in Women?" Annals of the Rheumatic Diseases, Vol. 56, No. 7, 1997, pp. 432-434. doi:10.1136/ard.56.7.432

[16] J. A. Martin, T. D. Brown, A. D. Heiner and J. A. Buckwalter, "Chondrocyte Senescence, Joint Loading and Osteoarthritis," Clinical Orthopaedics and Related Research, Vol. 427, 2004, S96-S103. doi:10.1097/01.blo.0000143818.74887.b1

[17] F. Guilak, B. Fermor, F. J. Keefe, V. B. Kraus, S. A. Olson, D. S. Pisetsky, et al., "The Role of Biomechanics and Inflammation in Cartilage Injury and Repair," Clinical Orthopaedics and Related Research, Vol. 423, 2004, pp. 17-26. doi:10.1097/01.blo.0000131233.83640.91

[18] D. R. Carter, G. S. Beaupre, M. Wong, R. L. Smith, T. P. Andriachhi and D. J. Schurman, "The Mechanobiology of Articular Cartilage Development and Degeneration," Clinical Orthopaedics and Related Research, Vol. 427, 2004, S69-S77. doi:10.1097/01.blo.0000144970.05107.7e

[19] L. Sharma, J. Song, D. T. Felson, S. Cahue, E. Shamiyeh and D. D. Dunlop, "The Role of Knee Alignment in Disease Progression and Functional Decline in Knee Osteoarthritis," The Journal of the American Medical Association, Vol. 286, No. 2, 2001, pp. 188-195. doi:10.1001/jama.286.2.188

[20] W. Zhang, "Editorial, Risk Factors of Knee Osteoarthritis -Excellent Evidence but Little Has Been Done," Osteoarthritis and Cartilage, Vol. 18, No. 1, 2009, pp. 1-2. doi:10.1016/j.joca.2009.07.013

[21] S. Kurtz, K. Ong, E. Lau, F. Mowat and M. Halpern, "Projections of Primary and Revision Hip and Knee Arthroplasty in the United States from 2005 to 2030," The Journal of Bone \& Joint Surgery, Vol. 89, No. 4, 2007, pp. 780-785. doi:10.2106/JBJS.F.00222

[22] J. H. Kellgren and J. S. Lawrence, "The Epidemiology of Chronic Rheumatism," In: J. H. Kellgren, Ed., Atlas of Standard Radiographs of Arthritis, FA Davis, Philadelphia, 1963, pp. 10-11.

[23] E. M. Hertogh, E. M. Monninkhof, E. G. Schouten, P. H. Peeters and A. J. Schuit, "Validity of the Modified Baecke Questionnaire: A Comparison with Energy Expenditure according to the Double Labeled Water Method," International Journal of Behavioral Nutrition and Physical Activity, Vol. 5, No. 5, 2008, p. 30. doi:10.1186/1479-5868-5-30 
[24] J. J. Bekkers, T. S. de Windt, N. H. Raijmakers, W. A. Dhert and D. F. Saris, "Validation of the Knee Injury and Osteoarthritis Outcome Score (KOOS) for Treatment of Focual Cartilage Lesions," Osteoarthritis and Cartilage, Vol. 17, No. 11, 2009, pp. 1434-1439. doi:10.1016/i.joca.2009.04.019

[25] H. G. Burger, E. C. Dudley, J. L. Hopper, J. M. Shelley, A. Green, A. Smith, et al., "The Endrocrinology of the Menopausal Transition: A Cross-Sectional Study of a Population-Based Sample," The Journal of Clinical Endocrinology and Metabolism, Vol. 80, No. 12, 1995, pp. 3537-3545. doi:10.1210/jc.80.12.3537

[26] J. Hee, J. MacNaughton, M. Bangah and H. G. Burger, "Perimenopausal Patterns of Gonadotrophins, Immunoreactive Inhibin, Estradiol and Progesterone," Maturitas, Vol. 18, No. 1, 1993, pp. 9-20. doi:10.1016/0378-5122(93)90026-E
[27] B. M. de Klerk, D. Schiphof, F. P. Groeneveld, B. W. Koes, G. J. van Osch, J. B. Meurs, et al., "No Clear Association between Female Hormonal Aspects and Osteoarthritis of the Hand, Hip and Knee: A Systematic Review," Rheumatology, Vol. 48, No. 9, 2009, pp. 11601165. doi:10.1093/rheumatology/kep194

[28] M. F. Sowers, C. F. Karvonen-Gutierrez, M. Yosef, M. Jannausch, Y. Jiang, P. Garnero, et al., "Longitudinal Changes of Serum COMP and Urinary CTX-II Predict X-Ray Defined Knee Osteoarthritis Severity and Stiffness in Women," Osteoarthritis and Cartilage, Vol. 17, No. 12, 2009, pp. 1609-1614. doi:10.1016/j.joca.2009.06.001

[29] R. Cerejo, D. D. Dunlop, S. Cahue, D. Channin, J. Song and L. Sharma, "The Influence of Alignment on Risk of Knee Osteoarthritis Progression according to Baseline Stage of Disease," Arthritis \& Rheumatism, Vol. 46, No. 10, 2002, pp. 2632-2636. doi:10.1002/art.10530 